\title{
On the Darboux integrability of a cubic CRN model in $\mathbb{R}^{5}$
}

\author{
Antoni Ferragut, Claudia Valls
}

\begin{abstract}
We study the Darboux integrability of a differential system in $\mathbb{R}^{5}$ with parameters coming from a chemical reaction model. In particular, we find all its Darboux polynomials and exponential factors and we prove that it is not Darboux integrable.
\end{abstract}

Keywords. Darboux polynomial; exponential factor; Darboux integrability; chemical reaction network

\section{Introduction and statement of the main result}

Consider an $n$-dimensional polynomial differential system of degree $d \in \mathbb{N}$

$$
\dot{\mathbf{x}}=P(\mathbf{x}), \quad \mathbf{x} \in \mathbb{R}^{n},
$$

where $P(\mathbf{x})=\left(P_{1}(\mathbf{x}), \ldots, P_{n}(\mathbf{x})\right), P_{i} \in \mathbb{C}[\mathbf{x}]$, and the dot denotes derivative with respect to the independent variable $t$.

A function $H(\mathbf{x})$ is a first integral of system (1.1) if it is continuous and defined in a full Lebesgue measure subset $\Omega \subseteq \mathbb{R}^{n}$, is not locally constant on any positive Lebesgue measure subset of $\Omega$ and moreover is constant along each orbit in $\Omega$ of system (1.1). If $H$ is $\mathcal{C}^{1}$ and we name $\mathcal{X}$ the vector field associated to system (1.1), then we have

$$
\mathcal{X}(H)=P_{1} \frac{\partial H}{\partial x_{1}}+\cdots+P_{n} \frac{\partial H}{\partial x_{n}}=0 .
$$

A. Ferragut: Institut Universitari de Matemàtiques i Aplicacions de Castelló (IMAC) and Departament de Matemàtiques; Universitat Jaume I, Edifici TI (ESTEC), Av. de Vicent Sos Baynat, s/n, Campus del Riu Sec, 12071 Castelló de la Plana, Spain; e-mail: ferragut@uji.es

C. Valls: Departamento de Matemática, Instituto Superior Técnico, Universidade Técnica de Lisboa, Av. Rovisco Pais, 1049-001, Lisboa, Portugal; e-mail: cvalls@ math.ist.utl.pt

Mathematics Subject Classification (2010): 34C05, 34A34, 34C14 
System (1.1) is $\mathcal{C}^{k}$-completely integrable in $\Omega$ if it has $n-1$ functionally independent $C^{k}$ first integrals in $\Omega$. Recall that $k$ functions $H_{1}(\mathbf{x}), \ldots, H_{k}(\mathbf{x})$ are functionally independent in $\Omega$ if the matrix of gradients $\left(\nabla H_{1}, \ldots, \nabla H_{k}\right)$ has rank $k$ in a full Lebesgue measure subset of $\Omega$.

For an $n$-dimensional system of differential equations the existence of some first integrals reduces the complexity of its dynamics and the existence of $n-1$ functionally independent first integrals solves completely the problem (at least theoretically) of determining its phase portrait. In general for a given differential system it is a difficult problem to determine the existence or non-existence of first integrals.

During recent years the interest in the study of the integrability of differential equations has attracted much attention from the mathematical community. Darboux theory of integrability plays a central role in the integrability of the polynomial differential systems since it gives a sufficient condition for the integrability inside a wide family of functions. We highlight that it works for real or complex polynomial differential systems and that the study of complex algebraic solutions is necessary for obtaining all the real first integrals of a real polynomial differential system.

A Darboux polynomial of (1.1) is a polynomial $f \in \mathbb{C}[\mathbf{x}]$ such that

$$
\mathcal{X}(f)=P_{1} \frac{\partial f}{\partial x_{1}}+\cdots+P_{n} \frac{\partial f}{\partial x_{n}}=k f
$$

where $\mathbf{x}=\left(x_{1}, \ldots, x_{n}\right)$ and $k \in \mathbb{C}[\mathbf{x}]$, which is called the cofactor of $f$, has degree at most $d-1$, where $d=\max \left\{\operatorname{deg} P_{1}, \ldots, \operatorname{deg} P_{n}\right\}$ is the degree of system (1.1). An invariant algebraic surface is a surface given by $f=0$. Note that it is invariant by the dynamics in the sense that if a trajectory starts on the surface it does not leave it.

An exponential factor of (1.1) is a function $F=\exp (g / f)$, with $f, g \in \mathbb{C}[\mathbf{x}]$, such that

$$
\mathcal{X}(F)=P_{1} \frac{\partial F}{\partial x_{1}}+\cdots+P_{n} \frac{\partial F}{\partial x_{n}}=L F,
$$

where $L \in \mathbb{C}[\mathbf{x}]$, which is called the cofactor of $F$, has degree at most $d-1$. It is widely known that in this case $f$ is a Darboux polynomial of (1.1) and that $\mathcal{X}(g)=k g+L f$, where $k$ is the cofactor of $f$.

A Darboux first integral $H$ has the form

$$
H=f_{1}^{\lambda_{1}} \cdots f_{p}^{\lambda_{p}} F_{1}^{\mu_{1}} \cdots F_{q}^{\mu_{q}}
$$


where $f_{1}, \ldots, f_{p}$ are Darboux polynomials, $F_{1}, \ldots, F_{q}$ are exponential factors and $\lambda_{i}, \mu_{j}$ are complex numbers, for $i=1, \ldots, p$ and $j=1, \ldots, q$.

The following result, proved in [7] explains how to find Darboux first integrals.

Proposition 1.1. Assume that a polynomial differential system of degree $m$ admits $p$ Darboux polynomials $f_{i}$ with cofactors $k_{i}, i=1, \ldots, p$, and q exponential factors $\exp \left(g_{j} / h_{j}\right)$ with cofactors $L_{j}, j=1, \ldots, q$. Then, there exist $\lambda_{i}, \mu_{j} \in \mathbb{C}$ not all zero such that

$$
\sum_{i=1}^{p} \lambda_{i} k_{i}+\sum_{j=1}^{q} \mu_{j} L_{j}=0
$$

if and only if the function given in (1.2) is a Darboux first integral of the polynomial differential system.

The Darboux theory of integrability relates the number of Darboux polynomials and exponentials factors of the differential system with the existence of a Darboux first integral, see for example [11]. We recall that a Darboux first integral is a product of complex powers of Darboux polynomials and exponential factors.

The main aim in this paper is to study the Darboux integrability of a cubic differential system that belongs to $\mathbb{R}^{5}$ and has an important contribution in Chemical Reaction Network Theory (CRNT). A chemical reaction network $\mathcal{N}=(\mathcal{S}, \mathcal{C}, \mathcal{R})$ is defined as a set of species $\mathcal{S}$, a set of complexes $\mathcal{C}$ and a set of reactions $\mathcal{R}$ between complexes; each complex is a combination of species. It is here assumed that a reaction occurs according to mass-action kinetics, that is, at a rate proportional to the product of the species concentrations in the reactant or source complex. The set of reactions together with a rate vector give rise to a polynomial system of ordinary differential equations. We refer the reader to $[8,9,10]$ for more information about CRNT. For a concrete system of chemical reactions the parameter and state spaces are typically high-dimensional and one uses numerical methods to analyze the solutions. Due to high computational complexity this can be done only for a small set of values of system's parameters. Thus instead of studying quantitative aspects of the dynamics, recently there has been an increasing interest in studying qualitative properties of the CRN. For example in $[1,2,3,4,5,6]$ the authors considered the question of existence of single versus multiple steady states (also referred to as multistationary). The existence of first integrals of a polynomial differential system describing a CRN often provides essential 
qualitative information (the level sets are invariant under the flow) about the solution or can be used to reduce the dimension of the total state space. Since the computation of nonlinear conservation laws (first integrals) is highly nontrivial, most of the known results related to the CRN dynamics provide only linear first integrals.

In this paper, our purpose is to show, by following an example (see system (1.4) below), how to apply the Darboux theory of integrability to obtain nontrivial and nonlinear algebraic and Darboux first integrals. Indeed, we consider the following reaction network appearing in [10]:

$$
A+2 B \rightarrow D \rightarrow A+C, \quad C+D \rightarrow E \rightarrow A+B
$$

By employing the common assumption that reaction rates are of mass-action type, the concentrations change with time according to the following system of ordinary differential equations:

$$
\begin{aligned}
& \dot{x}_{1}=-c_{1} x_{1} x_{2}^{2}+c_{2} x_{4}+c_{4} x_{5}, \\
& \dot{x}_{2}=-2 c_{1} x_{1} x_{2}^{2}+c_{4} x_{5}, \\
& \dot{x}_{3}=c_{2} x_{4}-c_{3} x_{3} x_{4}, \\
& \dot{x}_{4}=c_{1} x_{1} x_{2}^{2}-c_{2} x_{4}-c_{3} x_{3} x_{4}, \\
& \dot{x}_{5}=c_{3} x_{3} x_{4}-c_{4} x_{5},
\end{aligned}
$$

where $c_{1}, c_{2}, c_{3}, c_{4}$ are positive constants. This is a good example to work with since the system belongs to $\mathbb{R}^{5}$, it is cubic and some nice objects are found, such as six exponential factors and a Darboux first integral. In [10] the positive steady-state solutions of this system are studied. Here we go deeper in the study of this system by studying its Darboux integrability. We believe that the techniques used in this paper, such as reduction of one dimension of the system, can be used for studying other CRN systems and, in general, polynomial differential systems of high dimension.

We shall deal in this paper with this differential system. We shall study its Darboux integrability by characterizing its Darboux polynomials and exponential factors. In the following theorem, which is our main result, we prove that there just exist two Darboux first integrals (one polynomial and one Darboux), one invariant algebraic surface of degree one and six exponential factors. As far as we know in all papers dealing with CRN models, the authors search only linear first integrals. More complicated first integrals demand a 
deeper study, and in general, are very difficult to detect if the system is not Hamiltonian, which is the case. Note that we are finding a first integral (which is not linear) and one invariant algebraic surface, i.e., two new invariant objects which provide some light in the reduction of the dimension of the system and on its qualitative behavior.

Theorem 1.2. The following results hold for system (1.4).

(a) The unique irreducible polynomial first integral is $H_{1}=x_{1}+x_{4}+x_{5}$. Any other polynomial first integral is a polynomial function of $H_{1}$.

(b) The unique irreducible Darboux polynomial is $F=c_{2}-c_{3} x_{3}$. It has cofactor $k=-c_{3} x_{4}$.

(c) It has six exponential factors: $F_{1}=e^{x_{3}}, F_{2}=e^{x_{2}-2 x_{1}}, F_{3}=e^{x_{1}+x_{4}}, F_{4}=$ $e^{\left(x_{2}-2 x_{1}\right)^{2}}, F_{5}=e^{\left(2 x_{1}-x_{2}\right)\left(x_{1}-x_{3}+x_{4}\right)}$ and $F_{6}=e^{\left(x_{1}-x_{3}+x_{4}\right)^{2}}$. If $e^{g / h}$ is another exponential factor, then $h \in \mathbb{C}\left[H_{1}\right]$ and

$$
\begin{aligned}
g\left(x_{1}, x_{2}, x_{3}, x_{4}\right)= & a_{1} x_{3}+a_{2}\left(x_{2}-2 x_{1}\right)+a_{3}\left(x_{1}+x_{4}\right)+a_{4}\left(x_{2}-2 x_{1}\right)^{2} \\
& +a_{5}\left(x_{2}-2 x_{1}\right)\left(x_{1}-x_{3}+x_{4}\right)+a_{6}\left(x_{1}-x_{3}+x_{4}\right)^{2}
\end{aligned}
$$

with $a_{i} \in \mathbb{C}, i=1, \ldots, 6$.

(d) It has the (non-rational) Darboux first integral

$$
H_{2}=F^{3 c_{2} / c_{3}} e^{-\left(x_{1}+x_{4}\right)} e^{-\left(x_{2}-2 x_{1}\right)} e^{x_{3}}=F^{3 c_{2} / c_{3}} e^{x_{1}-x_{2}+x_{3}-x_{4}} .
$$

(e) It is not Darboux completely integrable.

As far as we know, in the papers about CRN only linear first integrals are searched, since more complicated first integrals demand a deeper study. Our aim in this paper is to provide an example where we find additional (not linear) first integrals in order to give some light in the reduction of the dimension of the system.

In order for the reader to follow the proofs of the paper that sometimes demand large expressions, we have added an appendix with a worksheet of mathematica with the computations that solve the PDE appearing in the paper. 


\section{Proof of the Theorem 1.2}

Statement (e) follows immediately from statements (a)-(d), since there is no way to construct two additional Darboux first integrals functionally independent of $H_{1}, H_{2}$. This assertion follows because there is no non-zero linear combination of the cofactors of the Darboux polynomials and exponentials factors of the system equal to zero. In particular, it is clear that the system has not rational first integrals. Hence, we need to prove only statements (a), (b), (c) and (d). We shall prove them separately.

\subsection{Proof of statement (a)}

Straightforward computations show that $H_{1}$ is a first integral of (1.4). The restriction of system (1.4) to $H_{1}=h$ is the differential system

$$
\begin{aligned}
& \dot{x}_{1}=-c_{1} x_{1} x_{2}^{2}-c_{4} x_{1}+\left(c_{2}-c_{4}\right) x_{4}+c_{4} h, \\
& \dot{x}_{2}=-2 c_{1} x_{1} x_{2}^{2}-c_{4} x_{1}-c_{4} x_{4}+c_{4} h, \\
& \dot{x}_{3}=\left(c_{2}-c_{3} x_{3}\right) x_{4}, \\
& \dot{x}_{4}=c_{1} x_{1} x_{2}^{2}-c_{2} x_{4}-c_{3} x_{3} x_{4} .
\end{aligned}
$$

Let $\mathcal{Y}_{h}$ be the corresponding vector field. For simplicity we shall write $\mathcal{Y}$ instead of $\mathcal{Y}_{h}$ because there is no possible confusion. Next lemma shows that (2.6) has no polynomial first integrals.

Lemma 2.1. System (2.6) has no polynomial first integrals.

Proof. Let $g\left(x_{1}, x_{2}, x_{3}, x_{4}\right)$ be a polynomial first integral of degree $m \in \mathbb{N}$ of system (2.6). We write $g=\sum_{i=1}^{m} g_{i}\left(x_{1}, x_{2}, x_{3}, x_{4}\right)$, where $g_{i}$ is a homogeneous polynomial of degree $i$, with $g_{m} \not \equiv 0$. The differential equation corresponding to the terms of degree $m+2$ of $\mathcal{Y}(g)=0$ is

$$
-c_{1} x_{1} x_{2}^{2}\left(\frac{\partial g_{m}}{\partial x_{1}}+2 \frac{\partial g_{m}}{\partial x_{2}}-\frac{\partial g_{m}}{\partial x_{4}}\right)=0,
$$

from which we obtain $g_{m}\left(x_{1}, x_{2}, x_{3}, x_{4}\right)=g_{m}\left(x_{3}, X_{1}, X_{2}\right)$, where we have introduced the variables $\left(X_{1}, X_{2}\right)=\left(x_{2}-2 x_{1}, x_{1}+x_{4}\right)$. Indeed we shall prove in a while that $g_{m}=g_{m}\left(X_{1}, X_{3}\right)$, where $X_{3}=X_{2}-x_{3}$.

Concerning the terms of degree $m+1$ we have the differential equation

$$
-c_{1} x_{1} x_{2}^{2}\left(\frac{\partial g_{m-1}}{\partial x_{1}}+2 \frac{\partial g_{m-1}}{\partial x_{2}}-\frac{\partial g_{m-1}}{\partial x_{4}}\right)-c_{3} x_{3} x_{4}\left(\frac{\partial g_{m}}{\partial x_{3}}+\frac{\partial g_{m}}{\partial x_{4}}\right)=0,
$$


from which we get

$$
\begin{aligned}
g_{m-1}= & \frac{c_{3} x_{3}}{c_{1}\left(x_{2}-2 x_{1}\right)}\left(\frac{\partial g_{m}}{\partial x_{3}}\left(x_{3}, X_{1}, X_{2}\right)+\frac{\partial g_{m}}{\partial X_{2}}\left(x_{3}, X_{1}, X_{2}\right)\right) \times \\
& \left(\frac{x_{1}+x_{4}}{x_{2}-2 x_{1}} \log \frac{x_{2}}{4 x_{1}}+\frac{x_{2}+2 x_{4}}{2 x_{2}}\right)+\bar{g}_{m-1}\left(x_{3}, x_{2}-2 x_{1}, x_{1}+x_{4}\right),
\end{aligned}
$$

where $\bar{g}_{m-1}$ is an arbitrary function. Since the logarithm must be removed, we have

$$
g_{m}=g_{m}\left(X_{1}, X_{3}\right)
$$

where we have introduced $X_{3}=X_{2}-x_{3}$, as we stated above. Hence we get $g_{m-1}=$ $g_{m-1}\left(x_{3}, X_{1}, X_{2}\right)$. Next we deal with the differential equation corresponding to the terms of degree $m$. We obtain

$$
\begin{aligned}
g_{m-2}= & \frac{X_{2} \log \frac{x_{2}}{4 x_{1}}}{c_{1} X_{1}^{2}}\left(\left(2 c_{2}-c_{4}\right) \frac{\partial g_{m}}{\partial X_{1}}+\left(c_{2}+c_{4}\right) \frac{\partial g_{m}}{\partial X_{2}}+c_{3} x_{3}\left(\frac{\partial g_{m-1}}{\partial x_{3}}+\frac{\partial g_{m-1}}{\partial X_{2}}\right)\right) \\
& +G_{m-2}+\bar{g}_{m-2}\left(x_{3}, X_{1}, X_{2}\right),
\end{aligned}
$$

where $G_{m-2}$ is a rational function and $\bar{g}_{m-2}$ is an arbitrary function. We must remove the logarithm, hence a PDE must be solved. We obtain

$$
g_{m-1}\left(x_{3}, X_{1}, X_{2}\right)=-\frac{\left(c_{2}+c_{4}\right) \frac{\partial g_{m}}{\partial X_{3}}+\left(2 c_{2}-c_{4}\right) \frac{\partial g_{m}}{\partial X_{1}}}{c_{3}} \log x_{3}+\bar{g}_{m-1}\left(X_{1}, X_{3}\right) .
$$

A new logarithm appears. To remove it we must take

$$
g_{m}\left(X_{1}, X_{3}\right)=\left(\left(c_{2}+c_{4}\right) X_{1}+\left(c_{4}-2 c_{2}\right) X_{3}\right)^{m},
$$

and therefore $g_{m-1}\left(x_{3}, X_{1}, X_{2}\right)=g_{m-1}\left(X_{1}, X_{3}\right)$. Now back to the expression of $g_{m-2}$ we have

$$
g_{m-2}=\frac{3 c_{2} c_{4} m}{2 c_{1}} \frac{\left(\left(c_{2}+c_{4}\right) X_{1}+\left(c_{4}-2 c_{2}\right) X_{3}\right)^{m-1}}{x_{2}}+\bar{g}_{m-2}\left(x_{3}, X_{1}, X_{2}\right) .
$$

Since $g_{m-2}$ is to be a polynomial, $x_{2} \nmid\left(\left(c_{2}+c_{4}\right) X_{1}+\left(c_{4}-2 c_{2}\right) X_{3}\right)$ and $c_{i}>0$ for all $i$, we have $m=0$. Then $g$ is a constant and the lemma follows.

Remark 2.2. The sequence of resolution in the proof of Lemma 2.1 will be used later on for other purposes.

After Lemma 2.1 we can prove statement (a) of Theorem 1.2. Let $f$ be a polynomial first integral of (1.4) which is not a function of $H_{1}$. Note that in view of Lemma 2.1, 
when $H_{1}=h$, the restricted system has no polynomial first integrals, so a polynomial first integral $f$ if it exists, when $H_{1}=h$ must be zero. By the Nunstelletstaz theorem, there exists a power $j$ (which may be zero) so that $H_{1}-h$ must divide $f$, so we write $f=\left(H_{1}-h\right)^{j} \bar{H}$, where $j \in \mathbb{N} \cup\{0\}$ and $\left(H_{1}-h\right) \nmid \bar{H}$, with $\bar{H}$ a polynomial. Since $X(f)=0$, we have $X(\bar{H})=0$. Let $g=\left.\bar{H}\right|_{H_{1}=h} \not \equiv 0$. Then $\mathcal{Y}(g)=0$. By Lemma 2.1 we have $g \equiv 0$, which is a contradiction with the assumption $\left(H_{1}-h\right) \nmid \bar{H}$. Hence such $f$ cannot exist and therefore statement (a) of Theorem 1.2 follows.

\subsection{Proof of statement $(b)$}

We start the study of the Darboux polynomials of system (1.4) by simplifying the general expression of the cofactor of any Darboux polynomial.

Proposition 2.3. Let $f$ be a Darboux polynomial of degree $m \in \mathbb{N}$ of system (1.4) with cofactor $k$. Then $k=k_{0}+k_{1} x_{1}+k_{2} x_{2}+k_{3} x_{3}+k_{4} x_{4}+k_{5} x_{5}+k_{6} x_{2}^{2}+k_{7} x_{1} x_{2}$, where $k_{i} \in \mathbb{C}$. Moreover, $-k_{6} / c_{1},-k_{7} /\left(2 c_{1}\right) \in \mathbb{N} \cup\{0\}$.

Proof. We write the general (quadratic) cofactor $k \in \mathbb{C}\left[x_{1}, x_{2}, x_{3}, x_{4}, x_{5}\right]$ as

$$
\begin{aligned}
k= & k_{0}+k_{1} x_{1}+k_{2} x_{2}+k_{3} x_{3}+k_{4} x_{4}+k_{5} x_{5}+k_{6} x_{1}^{2}+k_{7} x_{1} x_{2}+k_{8} x_{1} x_{3} \\
& +k_{9} x_{1} x_{4}+k_{10} x_{1} x_{5}+k_{11} x_{2}^{2}+k_{12} x_{2} x_{3}+k_{13} x_{2} x_{4}+k_{14} x_{2} x_{5} \\
& +k_{15} x_{3}^{2}+k_{16} x_{3} x_{4}+k_{17} x_{3} x_{5}+k_{18} x_{4}^{2}+k_{19} x_{4} x_{5}+k_{20} x_{5}^{2} .
\end{aligned}
$$

Taking the homogeneous part of degree $m+1$ of the equation $\mathcal{X}(f)=k f$ and using the Euler theorem of homogeneous functions for $f_{m}$ we get the equation

$$
\begin{aligned}
-\left[k_{6} x_{1}^{2}\right. & +k_{7} x_{1} x_{2}+k_{8} x_{1} x_{3}+k_{9} x_{1} x_{4}+k_{10} x_{1} x_{5}+\left(c_{1} m+k_{11}\right) x_{2}^{2}+k_{12} x_{2} x_{3} \\
& +k_{13} x_{2} x_{4}+k_{14} x_{2} x_{5}+k_{15} x_{3}^{2}+k_{16} x_{3} x_{4}+k_{17} x_{3} x_{5}+k_{18} x_{4}^{2}+k_{19} x_{4} x_{5} \\
& \left.+k_{20} x_{5}^{2}\right] f_{m}+c_{1} x_{2}^{2}\left[\left(x_{2}-2 x_{1}\right) \frac{\partial f_{m}}{\partial x_{2}}+x_{3} \frac{\partial f_{m}}{\partial x_{3}}+\left(x_{1}+x_{4}\right) \frac{\partial f_{m}}{\partial x_{4}}+x_{5} \frac{\partial f_{m}}{\partial x_{5}}\right] \\
& =0 .
\end{aligned}
$$

The general solution of this equation is

$$
\begin{aligned}
f_{m}\left(x_{1}, x_{2}, x_{3}, x_{4}, x_{5}\right)=e^{\frac{x_{1} P_{1}}{2 c_{1} x_{2}\left(2 x_{1}-x_{2}\right)^{2}}} & x_{2}^{\frac{P_{2}}{4 c_{1}\left(2 x_{1}-x_{2}\right)^{2}}}\left(2 x_{1}-x_{2}\right)^{m+\frac{P_{3}}{4 c_{1}}} \\
& \times C_{m}\left(x_{1}, \frac{x_{3}}{2 x_{1}-x_{2}}, \frac{x_{1}+x_{4}}{2 x_{1}-x_{2}}, \frac{x_{5}}{2 x_{1}-x_{2}}\right),
\end{aligned}
$$


where

$$
\begin{aligned}
P_{1}= & 4 k_{20} x_{5}^{2}+4 k_{19} x_{4} x_{5}+4 k_{18} x_{4}^{2}+4 k_{17} x_{3} x_{5}+4 k_{16} x_{3} x_{4}+4 k_{15} x_{3}^{2} \\
& +2\left(k_{19}-k_{10}\right) x_{2} x_{5}+2\left(2 k_{18}-k_{9}\right) x_{2} x_{4}+2\left(k_{16}-k_{8}\right) x_{2} x_{3} \\
& +4 k_{10} x_{1} x_{5}+4 k_{9} x_{1} x_{4}+4 k_{8} x_{1} x_{3}+2\left(k_{9}-2 k_{6}\right) x_{1} x_{2}+4 k_{6} x_{1}^{2} \\
& +\left(k_{18}-k_{9}+k_{6}\right) x_{2}^{2} ; \\
P_{2}= & 4 k_{20} x_{5}^{2}+4 k_{19} x_{4} x_{5}+4 k_{18} x_{4}^{2}+4 k_{17} x_{3} x_{5}+4 k_{16} x_{3} x_{4}+4 k_{15} x_{3}^{2}+4 k_{14} x_{2} x_{5} \\
& +4 k_{13} x_{2} x_{4}+4 k_{12} x_{2} x_{3}+4\left(k_{9}-2 k_{7}-k_{6}\right) x_{1}^{2} \\
& +\left(-k_{18}+2 k_{13}+k_{9}-2 k_{7}-k_{6}\right) x_{2}^{2}+4\left(k_{19}-2 k_{14}\right) x_{1} x_{5}+8\left(k_{18}-k_{13}\right) x_{1} x_{4} \\
& +4\left(k_{16}-2 k_{12}\right) x_{1} x_{3}+4\left(k_{18}-k_{13}-k_{9}+2 k_{7}+k_{6}\right) x_{1} x_{2} ; \\
P_{3}= & k_{18}-2 k_{13}+4 k_{11}-k_{9}+2 k_{7}+k_{6} ;
\end{aligned}
$$

and $C_{m}$ is an arbitrary function. In order to get a polynomial the exponent of the exponential must be a constant and the exponents of $x_{2}$ and $2 x_{1}-x_{2}$ must be non-negative integers. Therefore we must take $k_{11}=-c_{1} n_{1}$ and $k_{7}=-2 c_{1} n_{2}$, where $n_{1}, n_{2} \in \mathbb{N} \cup\{0\}$, and $k_{6}=k_{8}=k_{9}=k_{10}=0, k_{12}=\cdots=k_{20}=0$. We get

$$
\begin{aligned}
& f_{m}\left(x_{1}, x_{2}, x_{3}, x_{4}, x_{5}\right) \\
& \quad=x_{2}^{n_{2}}\left(x_{2}-2 x_{1}\right)^{m-n_{1}-n_{2}} C_{m}\left(x_{1}, \frac{x_{3}}{x_{2}-2 x_{1}}, \frac{x_{1}+x_{4}}{x_{2}-2 x_{1}}, \frac{x_{5}}{x_{2}-2 x_{1}}\right) .
\end{aligned}
$$

Since this is to be a polynomial of degree $m$, we take

$$
f_{m}\left(x_{1}, x_{2}, x_{3}, x_{4}, x_{5}\right)=x_{1}^{n_{1}} x_{2}^{n_{2}}\left(x_{2}-2 x_{1}\right)^{m-n_{1}-n_{2}-n} P_{n}\left(x_{3}, x_{1}+x_{4}, x_{5}\right),
$$

where $P_{n}$ is a homogeneous polynomial of degree $n \in \mathbb{N} \cup\{0\}, n \leq m-n_{1}-n_{2}$. Renaming the coefficients of $k$, the proposition follows.

Lemma 2.4. The unique Darboux polynomial of degree one of system (1.4) is $c_{2}-c_{3} x_{3}$. Its cofactor is $k=-c_{3} x_{4}$.

Proof. It follows after easy computations.

Next lemma shows that there are no more Darboux polynomials, and thus it finishes the proof of statement (b) of Theorem 1.2.

Lemma 2.5. System (1.4) has no irreducible Darboux polynomials of degree greater than one. 
Proof. Let $\mathcal{F}$ be an irreducible Darboux polynomial of system (1.4) and let $f=\mathcal{F}_{H_{1}=h}$. We recall that $F=c_{2}-c_{3} x_{3}$ is a Darboux polynomial of system (2.6). Consider system (2.6) restricted to $F=0$ :

$$
\begin{aligned}
& \dot{x}_{1}=-c_{1} x_{1} x_{2}^{2}-c_{4} x_{1}+\left(c_{2}-c_{4}\right) x_{4}+c_{4} h, \\
& \dot{x}_{2}=-2 c_{1} x_{1} x_{2}^{2}-c_{4} x_{1}-c_{4} x_{4}+c_{4} h, \\
& \dot{x}_{4}=c_{1} x_{1} x_{2}^{2}-2 c_{2} x_{4} .
\end{aligned}
$$

Note that $f$ is an irreducible Darboux polynomial of system (2.6). Let $g$ be the Darboux polynomial of (2.7) corresponding to $f$ restricted to $F=0$; that is, $g=\left.f\right|_{F=0}$. Let $m \in \mathbb{N} \cup\{0\}$ be the degree of $g$. We have:

$$
\begin{aligned}
\left(-c_{1} x_{1} x_{2}^{2}-c_{4} x_{1}+\left(c_{2}-c_{4}\right) x_{4}+c_{4} h\right) \frac{\partial g}{\partial x_{1}} & \\
& +\left(-2 c_{1} x_{1} x_{2}^{2}-c_{4} x_{1}-c_{4} x_{4}+c_{4} h\right) \frac{\partial g}{\partial x_{2}}+\left(c_{1} x_{1} x_{2}^{2}-2 c_{2} x_{4}\right) \frac{\partial g}{\partial x_{4}} \\
& -\left(k_{0}+k_{1} x_{1}+k_{2} x_{2}+k_{4} x_{4}-c_{1} n_{1} x_{2}^{2}-2 c_{1} n_{2} x_{1} x_{2}\right) g=0 .
\end{aligned}
$$

The expression of the cofactor of $g$ can be deduced from Proposition 2.3. We write $g=$ $\sum_{i=0}^{m} g_{i}(x, y)$, with $g_{i}$ a homogeneous polynomial of degree $i, g_{m} \not \equiv 0$. We shall prove that $m=0$.

From (2.8), the equation of degree $m+2$ becomes, after canceling a common factor $c_{1} x_{2}$,

$$
-x_{1} x_{2}\left(\frac{\partial g_{m}}{\partial x_{1}}+2 \frac{\partial g_{m}}{\partial x_{2}}-\frac{\partial g_{m}}{\partial x_{4}}\right)+\left(n_{1} x_{2}+2 n_{2} x_{1}\right) g_{m}=0
$$

Then $g_{m}=x_{1}^{n_{1}} x_{2}^{n_{2}} \bar{g}_{m}\left(x_{2}-2 x_{1}, x_{1}+x_{4}\right)$, with $\bar{g}_{m}$ a homogeneous polynomial of degree $m-n_{1}-n_{2}$. The equation of degree $m+1$ of (2.8) is

$$
\begin{aligned}
-c_{1} x_{1} x_{2}^{2}\left(\frac{\partial g_{m-1}}{\partial x_{1}}+\right. & \left.2 \frac{\partial g_{m-1}}{\partial x_{2}}-\frac{\partial g_{m-1}}{\partial x_{4}}\right)+c_{1} x_{2}\left(n_{1} x_{2}+2 n_{2} x_{1}\right) g_{m-1} \\
& -\left(k_{1} x_{1}+k_{2} x_{2}+k_{4} x_{4}\right) x_{1}^{n_{1}} x_{2}^{n_{2}} \bar{g}_{m}\left(x_{2}-2 x_{1}, x_{1}+x_{4}\right)=0
\end{aligned}
$$

from which we obtain

$$
\begin{aligned}
g_{m-1}= & -\frac{2 k_{1} x_{1}-k_{1} x_{2}+k_{4} x_{2}+2 k_{4} x_{4}}{2 c_{1}\left(x_{2}-2 x_{1}\right)} x_{1}^{n_{1}} x_{2}^{n_{2}-1} \bar{g}_{m}\left(x_{2}-2 x_{1}, x_{1}+x_{4}\right) \\
& -\frac{\left(2 k_{2}-k_{4}\right) x_{1}-k_{2} x_{2}-k_{4} x_{4}}{c_{1}\left(x_{2}-2 x_{1}\right)^{2}} x_{1}^{n_{1}} x_{2}^{n_{2}} \bar{g}_{m}\left(x_{2}-2 x_{1}, x_{1}+x_{4}\right) \log \frac{x_{2}}{4 x_{1}} \\
& +x_{1}^{n_{1}} x_{2}^{n_{2}} \bar{g}_{m-1}\left(x_{2}-2 x_{1}, x_{1}+x_{4}\right),
\end{aligned}
$$


where $\bar{g}_{m-1}$ is a homogeneous polynomial of degree $m-n_{1}-n_{2}-1$. Since the logarithm must be removed, we have $k_{2}=k_{4}=0$. Hence

$$
\begin{aligned}
g_{m-1}\left(x_{1}, x_{2}, x_{4}\right)=\frac{k_{1}}{2 c_{1}} x_{1}^{n_{1}} x_{2}^{n_{2}-1} \bar{g}_{m}\left(x_{2}-2 x_{1},\right. & \left.x_{1}+x_{4}\right) \\
& +x_{1}^{n_{1}} x_{2}^{n_{2}} \bar{g}_{m-1}\left(x_{2}-2 x_{1}, x_{1}+x_{4}\right) .
\end{aligned}
$$

The equation of degree $m$ of (2.8) is

$$
\begin{gathered}
-c_{1} x_{1} x_{2}^{2}\left(\frac{\partial g_{m-2}}{\partial x_{1}}+2 \frac{\partial g_{m-2}}{\partial x_{2}}-\frac{\partial g_{m-2}}{\partial x_{4}}\right)+c_{1} x_{2}\left(n_{1} x_{2}+2 n_{2} x_{1}\right) g_{m-2} \\
+\left(\left(c_{2}-c_{4}\right) x_{4}-c_{4} x_{1}\right) \frac{\partial g_{m}}{\partial x_{1}}-c_{4}\left(x_{1}+x_{4}\right) \frac{\partial g_{m}}{\partial x_{2}}-2 c_{2} x_{4} \frac{\partial g_{m}}{\partial x_{4}}-k_{0} g_{m}-k_{1} x_{1} g_{m-1}=0 .
\end{gathered}
$$

We obtain

$$
\begin{aligned}
g_{m-2}\left(x_{1}, x_{2}, x_{4}\right)= & \frac{x_{1}^{n_{1}} x_{2}^{n_{2}}}{c_{1}\left(x_{2}-2 x_{1}\right)^{3}} E_{m-2}\left(\bar{g}_{m}\right) \log \frac{x_{2}}{x_{1}} \\
& \quad+\frac{x_{1}^{n_{1}-1} x_{2}^{n_{2}-2}}{c_{1}^{2}\left(x_{2}-2 x_{1}\right)^{2}} P_{m-2}+x_{1}^{n_{1}} x_{2}^{n_{2}} \bar{g}_{m-2}\left(x_{2}-2 x_{1}, x_{1}+x_{4}\right),
\end{aligned}
$$

where $\bar{g}_{m-2}$ is a homogeneous polynomial of degree $m-n_{1}-n_{2}-2, P_{m-2}$ is a homogeneous polynomial and $E_{m-2}\left(\bar{g}_{m}\right)=0$ is the following PDE with unknown $\bar{g}_{m}$ :

$$
\begin{aligned}
-\left(c_{4}\left(-4 n_{1}+n_{2}\right) X_{1}+k_{0} X_{2}\right. & \left.+c_{2} n_{1}\left(4 X_{1}+X_{2}\right)\right) \bar{g}_{m} \\
& -\left(c_{2}+c_{4}\right) X_{1} X_{2} \frac{\partial \bar{g}_{m}}{\partial X_{1}}+\left(2 c_{2}-c_{4}\right) X_{1} X_{2} \frac{\partial \bar{g}_{m}}{\partial X_{2}}=0
\end{aligned}
$$

where we have written $X_{1}=x_{2}-2 x_{1}$ and $X_{2}=x_{1}+x_{4}$ for simplicity. This (homogeneous) equation has the solution

$$
\bar{g}_{m}=X_{1}^{n_{4}} X_{2}^{n_{3}}\left(c_{2}\left(2 X_{1}-X_{2}\right)-c_{4}\left(X_{1}+X_{2}\right)\right)^{m-n_{1}-n_{2}-n_{3}-n_{4}}
$$

where $n_{3}, n_{4} \in \mathbb{N} \cup\{0\}$ are such that $m-\sum_{i=1}^{4} n_{i} \geq 0, k_{0}+c_{2} n_{1}+\left(c_{2}+c_{4}\right) n_{4}=0$ and

$$
c_{4} n_{2}+4\left(c_{2}-c_{4}\right) n_{1}+\left(2 c_{2}-c_{4}\right) n_{3}=0
$$

Since the logarithm in the expression of $g_{m-2}$ above must be removed, we fix this expression for $\bar{g}_{m}$ to cancel $E_{m-2}$. 
We note that this expression holds for $c_{4} \neq 2 c_{2}$. The case $c_{4}=2 c_{2}$ will be considered later on in this proof. The equation of degree $m-1$ of (2.8) is

$$
\begin{aligned}
-c_{1} x_{1} & x_{2}^{2} \\
& \left(\frac{\partial g_{m-3}}{\partial x_{1}}+2 \frac{\partial g_{m-3}}{\partial x_{2}}-\frac{\partial g_{m-3}}{\partial x_{4}}\right)+c_{1} x_{2}\left(n_{1} x_{2}+2 n_{2} x_{1}\right) g_{m-3} \\
& +\left(\left(c_{2}-c_{4}\right) x_{4}-c_{4} x_{1}\right) \frac{\partial g_{m-1}}{\partial x_{1}}-c_{4}\left(x_{1}+x_{4}\right) \frac{\partial g_{m-1}}{\partial x_{2}}-2 c_{2} x_{4} \frac{\partial g_{m-1}}{\partial x_{4}} \\
& +\left(c_{2} n_{1}+\left(c_{2}+c_{4}\right) n_{4}\right) g_{m-1}-k_{1} x_{1} g_{m-2}+c_{4} h\left(\frac{\partial g_{m}}{\partial x_{1}}+\frac{\partial g_{m}}{\partial x_{2}}\right)=0
\end{aligned}
$$

We do not write the expression of $g_{m-3}$ because it is too long. In this expression there is a logarithm that must be removed. Its coefficient provides a PDE with unknown $\bar{g}_{m-1}$ :

$$
\begin{aligned}
& -\frac{1}{4 c_{1}} X_{1}^{n_{4}-1} X_{2}^{n_{3}-1}\left(c_{2}\left(2 X_{1}-X_{2}\right)-c_{4}\left(X_{1}+X_{2}\right)\right)^{m-n_{1}-n_{2}-n_{3}-n_{4}-1} \times \\
& \quad\left[6 c_{2}^{2} k_{1}\left(2 n_{1}+n_{3}\right) X_{1}^{2}\left(2 X_{1}-X_{2}\right)+c_{4}^{2}\left(X_{1}+X_{2}\right)\left(k_{1}\left(2+12 n_{1}-3 n_{2}+3 n_{3}\right) X_{1}^{2}\right.\right. \\
& \left.\quad+4 c_{1} h X_{2}\left(-4 n_{1} X_{1}+n_{2} X_{1}-n_{3} X_{1}+n_{4} X_{2}\right)\right)+c_{2} c_{4}\left(-k_{1} X_{1}^{2}\left(2\left(2+18 n_{1}-3 n_{2}+6 n_{3}\right) X_{1}\right.\right. \\
& \left.\quad+\left(-2+3 n_{2}+3 n_{3}\right) X_{2}\right)+4 c_{1} h X_{2}\left(2 n_{3} X_{1}^{2}-2 n_{2} X_{1}\left(X_{1}-2 X_{2}\right)+n_{1} X_{1}\left(8 X_{1}-X_{2}\right)\right. \\
& \left.\left.\left.\quad-3 m X_{1} X_{2}+2 n_{3} X_{1} X_{2}+n_{4} X_{1} X_{2}+n_{4} X_{2}^{2}\right)\right)\right]-\left(c_{2}+c_{4}\right) X_{1} X_{2} \frac{\partial \bar{g}_{m-1}}{\partial X_{1}} \\
& \quad+\left(c_{4}-2 c_{2}\right) X_{1} X_{2} \frac{\partial \bar{g}_{m-1}}{\partial X_{2}}+\left(\left(-4 c_{2} n_{1}+4 c_{4} n_{1}-c_{4} n_{2}\right) X_{1}+\left(c_{2}+c_{4}\right) n_{4} X_{2}\right) \bar{g}_{m-1}=0 .
\end{aligned}
$$

From this new differential equation we can obtain the expression of $\bar{g}_{m-1}$. To facilitate the computations we use the Euler Theorem of homogeneous functions:

$$
\begin{aligned}
X_{1}^{n_{4}-1} & X_{2}^{n_{3}-1}\left(c_{2}\left(2 X_{1}-X_{2}\right)-c_{4}\left(X_{1}+X_{2}\right)\right)^{m-n_{1}-n_{2}-n_{3}-n_{4}-1}\left[6 c_{2}^{2} k_{1}\left(2 n_{1}+n_{3}\right) X_{1}^{2}\left(2 X_{1}-X_{2}\right)\right. \\
& +c_{4}^{2}\left(X_{1}+X_{2}\right)\left(k_{1}\left(2+12 n_{1}-3 n_{2}+3 n_{3}\right) X_{1}^{2}+4 c_{1} h X_{2}\left(-4 n_{1} X_{1}+n_{2} X_{1}\right.\right. \\
& \left.\left.-n_{3} X_{1}+n_{4} X_{2}\right)\right)+c_{2} c_{4}\left(-k_{1} X_{1}^{2}\left(2\left(2+18 n_{1}-3 n_{2}+6 n_{3}\right) X_{1}+\left(-2+3 n_{2} 3 n_{3}\right) X_{2}\right)\right. \\
& +4 c_{1} h X_{2}\left(2 n_{3} X_{1}^{2}-2 n_{2} X_{1}\left(X_{1}-2 X_{2}\right)+n_{1} X_{1}\left(8 X_{1}-X_{2}\right)-3 m X_{1} X_{2}+2 n_{3} X_{1} X_{2}\right. \\
& \left.\left.\left.+n_{4} X_{1} X_{2}+n_{4} X_{2}^{2}\right)\right)\right]+4 c_{1}\left(c_{4}\left(-\left(-1+m+3 n_{1}-2 n_{2}\right) X_{1}-n_{4} X_{2}\right)\right. \\
& \left.+c_{2}\left(2\left(-1+m+n_{1}-n_{2}\right) X_{1}-n_{4} X_{2}\right)\right) \bar{g}_{m-1}\left(X_{1}, X_{2}\right) \\
& +4 c_{1} X_{1}\left(c_{2}\left(-2 X_{1}+X_{2}\right)+c_{4}\left(X_{1}+X_{2}\right)\right) \frac{\partial \bar{g}_{m-1}}{\partial X_{1}}=0 .
\end{aligned}
$$

We can solve the homogeneous part of this PDE to obtain

$$
\begin{aligned}
& \bar{g}_{m-1}\left(X_{1}, X_{2}\right)=X_{1}^{n_{4}} \\
& \quad\left(c_{2}\left(2 X_{1}-X_{2}\right)-c_{4}\left(X_{1}+X_{2}\right)\right)^{\frac{c_{4}-c_{4}\left(m+3 n_{1}-2 n_{2}\right)+2 c_{2}\left(m+n_{1}-n_{2}-1\right)}{2 c_{2}-c_{4}}-n_{4}} \bar{G}_{m-1}\left(X_{2}\right),
\end{aligned}
$$

where $\bar{G}_{m-1}$ is an arbitrary function. Now replacing $\bar{G}_{m-1}\left(X_{2}\right)$ by $\bar{G}_{m-1}\left(X_{1}, X_{2}\right)$ and replacing $n_{3}$ by its value, that can be obtained from (2.9), we can compute the expression 
of $\bar{G}_{m-1}$ and therefore of $\bar{g}_{m-1}$. We obtain:

$$
\begin{aligned}
\bar{g}_{m-1}\left(X_{1}, X_{2}\right)= & X_{1}^{n_{4}-1}\left(c_{2}\left(2 X_{1}-X_{2}\right)-c_{4}\left(X_{1}+X_{2}\right)\right)^{c_{4}-\frac{c_{4}\left(m+3 n_{1}-2 n_{2}\right)+2 c_{2}\left(m+n_{1}-n_{2}-1\right)}{2 c_{2}-c_{4}}-n_{4}} \times \\
& {\left[c_{4} h n_{4} X_{2}^{\frac{c_{2}\left(2-4 n_{1}\right)+c_{4}\left(-1+4 n_{1}-n_{2}\right)}{2 c_{2}-c_{4}}}-\frac{c_{4} k_{1}}{2 c_{1}} X_{1} X_{2}^{\frac{-2 c_{2}+c_{4}-4 c_{2} n_{1}+4 c_{4} n_{1}-c_{4} n_{2}}{2 c_{2}-c_{4}}}\right.} \\
& +\frac{c_{2} c_{4} h}{c_{2}+c_{4}}\left[3 m+5 n_{1}-4 n_{2}-3 n_{4}\right] X_{2}^{-\frac{4 c_{2} n_{1}-4 c_{4} n_{1}+c_{4} n_{2}}{2 c_{2}-c_{4}}} \log \left(X_{1}\right) \\
& -\frac{3 c_{2} c_{4} h}{2 c_{2}^{2}+c_{2} c_{4}-c_{4}^{2}}\left[2 c_{2}\left(m+n_{1}-n_{2}-n_{4}\right)+c_{4}\left(-m-3 n_{1}+2 n_{2}+n_{4}\right)\right] \\
& X_{2}^{-\frac{4 c_{2} n_{1}-4 c_{4} n_{1}+c_{4} n_{2}}{2 c_{2}-c_{4}}} \log \left(c_{2}\left(2 X_{1}-X_{2}\right)-c_{4}\left(X_{1}+X_{2}\right)\right) \\
& \left.+C_{m-1} X_{2}^{-\frac{4 c_{2} n_{1}-4 c_{4} n_{1}+c_{4} n_{2}}{2 c_{2}-c_{4}}}\right],
\end{aligned}
$$

where $C_{m-1}$ is a constant. The coefficients of the logarithms must vanish in order to have a polynomial, so we have some conditions on the coefficients of the system and on the exponents: either $3\left(m-n_{4}\right)+5 n_{1}-4 n_{2}=0$ and $c_{2}+c_{4}=0$, or $m=n_{2} / 2+n_{4}$ and $n_{1}=n_{2} / 2$. The first case does not hold since $c_{2}, c_{4}>0$. Hence the second case is the only one to be considered. From (2.9) we get $\left(c_{4}-2 c_{2}\right)\left(n_{2}+n_{3}\right)=0$. Since we are assuming $c_{4} \neq 2 c_{2}$, we have $n_{2}=n_{3}=0$, and thus $n_{1}=0$ and $n_{4}=m$. After this, we have $g_{m}=\left(x_{1}+x_{4}\right)^{m}$ and

$$
\begin{aligned}
g_{m-1}= & \frac{X_{1}^{m-1}}{2 c_{1} X_{2}\left(2 c_{2} X_{1}-c_{4} X_{1}-c_{2} X_{2}-c_{4} X_{2}\right)\left(2 X_{1}+X_{2}-2 x_{4}\right)}\left(-2 c_{4} k_{1} X_{1}^{3}+4 c_{1} C_{m-1} X_{1}^{2} X_{2}\right. \\
& +2 c_{2} k_{1} X_{1}^{2} X_{2}-2 c_{4} k_{1} X_{1}^{2} X_{2}+2 c_{1} C_{m-1} X_{1} X_{2}^{2}-c_{2} k_{1} X_{1} X_{2}^{2}-c_{4} k_{1} X_{1} X_{2}^{2} \\
& \left.+4 c_{1} c_{4} h m X_{1} X_{2}^{2}+2 c_{1} c_{4} h m X_{2}^{3}+2 c_{4} k_{1} X_{1}^{2} x_{4}-4 c_{1} C_{m-1} X_{1} X_{2} x_{4}-4 c_{1} c_{4} h m X_{2}^{2} x_{4}\right) .
\end{aligned}
$$

To ensure that this is a polynomial, we must take $k_{1}=0$ (just dividing and equaling the remainder to zero). Now

$$
g_{m-1}=-X_{1}^{m-1} \frac{C_{m-1} X_{1}+c_{4} h m X_{2}}{c_{2}\left(-2 X_{1}+X_{2}\right)+c_{4}\left(X_{1}+X_{2}\right)},
$$

so we fix

$$
C_{m-1}=\frac{c_{4} h m\left(c_{4}-2 c_{2}\right)}{c_{2}+c_{4}}
$$

to finally obtain a polynomial. Now we have, for $g_{m-2}$,

$$
g_{m-2}=-\frac{c_{2} m}{2 c_{1} x_{2}}\left(x_{1}+x_{4}\right)^{m-1}+\bar{g}_{m-2}\left(x_{2}-2 x_{1}, x_{1}+x_{4}\right) .
$$

To obtain a polynomial we must take $m=0$.

Next we consider the case $c_{4}=2 c_{2}$. We start over and, with similar arguments, we obtain $k_{2}=k_{4}=0, k_{0}=-c_{2}\left(n_{1}+3 n_{4}\right), n_{2}=2 n_{1}$ and $n_{1}=m-n_{4}$ (there is no $n_{3}$ but we are keeping the previous notation). We have

$$
g_{m}=x_{1}^{m-n_{4}} x_{2}^{2\left(m-n_{4}\right)}\left(x_{2}-2 x_{1}\right)^{-2\left(m-n_{4}\right)}\left(x_{1}+x_{4}\right)^{n_{4}} .
$$


Then $n_{4}=m$. We also obtain

$$
\begin{aligned}
g_{m-1}= & -\frac{X_{1}^{m-1}}{6 c_{1} X_{2}^{2}\left(2 X_{1}+X_{2}-2 x_{4}\right)}\left[-4 k_{1} X_{1}^{3}-12 c_{1} C_{m-1} X_{1}^{2} X_{2}-2 k_{1} X_{1}^{2} X_{2}\right. \\
& -6 c_{1} C_{m-1} X_{1} X_{2}^{2}-3 k_{1} X_{1} X_{2}^{2}+8 c_{1} h m X_{1} X_{2}^{2}+4 c_{1} h m X_{2}^{3}+4 k_{1} X_{1}^{2} x_{4} \\
& \left.+12 c_{1} C_{m-1} X_{1} X_{2} x_{4}-8 c_{1} h m X_{2}^{2} x_{4}\right] .
\end{aligned}
$$

We need again $k_{1}=0$, and then

$$
g_{m-1}=-\frac{2}{3} h m X_{1}^{m-1}+\frac{C_{m-1}}{X_{2}} X_{1}^{m} .
$$

Hence $C_{m-1}=0$. Finally we have

$$
g_{m-2}=-\frac{c_{2} m}{2 c_{1} x_{2}}\left(x_{1}+x_{4}\right)^{m-1}+\bar{g}_{m-2}\left(x_{2}-2 x_{1}, x_{1}+x_{4}\right) .
$$

Again we must take $m=0$.

So we have proved that $m=0$; that is, $\operatorname{deg} g=0$. Thus $f$ restricted to $F=0$ is a constant. Write $f=c+F^{j} \bar{f}$, where $c$ is that constant, $j \in \mathbb{N}$ and $\bar{f}$ is a polynomial such that $F \nmid \bar{f}$. We note that $c \neq 0$ since $f$ is irreducible.

Taking into account this expression of $f$, we check the equation $\mathcal{Y}(f)=k f$, where $k$ is the cofactor of $f$ :

$$
F^{j}\left(\mathcal{Y}(\bar{f})-\left(j c_{3} x_{4}+k\right) \bar{f}\right)=c k .
$$

If $\Delta:=\mathcal{Y}(\bar{f})-\left(j c_{3} x_{4}+k\right) \bar{f} \equiv 0$ then, since $k \not \equiv 0$ from Lemma 2.1, we have $c=0$, which is a contradiction. Thus $\Delta \not \equiv 0$ and hence $F \mid k$, which implies that there exists $\alpha \in \mathbb{C} \backslash\{0\}$ such that $k=\alpha F$. Moreover equation (2.10) becomes $F^{j-1} \Delta=c \alpha$; hence $j=1$ and

$$
\mathcal{Y}(\bar{f})-\left(\alpha F+c_{3} x_{4}\right) \bar{f}=c \alpha .
$$

Applying the arguments in the proof of Lemma 2.1 and taking into account that $k$ is linear (from Proposition 2.3 and because $n_{1}=n_{2}=0$ ), we get that $\bar{f}$ is a (nonzero) constant, say $\bar{f}=\beta$. Hence $f=c+\beta F$. Since $c \neq 0$, this is a contradiction with Lemma 2.4.

\subsection{Proof of statement (c)}

We consider system (1.4) restricted to $H_{1}=h$; that is, we consider system (2.6). The following result characterizes the exponential factors of system (2.6) of the form $\exp (g)$, with $g \in \mathbb{C}\left[x_{1}, x_{2}, x_{3}, x_{4}\right]$.

Lemma 2.6. Let $\exp (g)$, with $g \in \mathbb{C}\left[x_{1}, x_{2}, x_{3}, x_{4}\right]$, be an exponential factor of system (2.6). Then $g$ is a linear combination of $x_{3}, x_{2}-2 x_{1}, x_{1}+x_{4},\left(x_{2}-2 x_{1}\right)^{2},\left(x_{2}-\right.$ $\left.2 x_{1}\right)\left(x_{1}-x_{3}+x_{4}\right)$ and $\left(x_{1}-x_{3}+x_{4}\right)^{2}$. 
Proof. Since $\exp (g)$ is an exponential factor of system (2.6), $g$ satisfies

$$
\begin{aligned}
\mathcal{Y}(g)=k= & k_{0}+k_{1} x_{1}+k_{2} x_{2}+k_{3} x_{3}+k_{4} x_{4}+k_{5} x_{1}^{2}+k_{6} x_{1} x_{2}+k_{7} x_{1} x_{3}+k_{8} x_{1} x_{4} \\
& +k_{9} x_{2}^{2}+k_{10} x_{2} x_{3}+k_{11} x_{2} x_{4}+k_{12} x_{3}^{2}+k_{13} x_{3} x_{4}+k_{14} x_{4}^{2},
\end{aligned}
$$

where $k$ is its cofactor and the $k_{i}$ are complex numbers. Assume that $g$ is a polynomial of degree $m \in \mathbb{N}$, with $m \geq 3$. We write it as sum of its homogeneous parts $g=\sum_{i=1}^{m} g_{i}\left(x_{1}, x_{2}, x_{3}, x_{4}\right)$, where $g_{i}$ is a homogeneous polynomial of degree $i$ and $g_{m} \not \equiv 0$. The right hand side of (2.11) has degree two, hence its left hand side must also have degree two. Since $m \geq 3$, the computation of $g_{m}, g_{m-1}$ and $g_{m-2}$ follows in the same way as the proof of Lemma 2.1. Therefore we get $m=0$, which is a contradiction. Hence $m \leq 2$. Easy computations show that $g$ is a linear combination of $x_{3}, x_{2}-2 x_{1}$, $x_{1}+x_{4},\left(x_{2}-2 x_{1}\right)^{2},\left(x_{2}-2 x_{1}\right)\left(x_{1}-x_{3}+x_{4}\right)$ and $\left(x_{1}-x_{3}+x_{4}\right)^{2}$.

Remark 2.7. In particular, the functions appearing in statement (c) of Theorem 1.2 are exponential factors.

In view of Lemma 2.6, if $E=\exp (g)$ is an exponential factor of system (2.6), then $g$ writes as (1.5) and the cofactor of $E$ has the form

$$
L=L_{0}+c_{4} L_{1} H_{1},
$$

where

$$
\begin{aligned}
L_{0} & =\left(a_{2}-a_{3}\right) c_{4} x_{1}+\left(\left(a_{1}-2 a_{2}\right) c_{2}+\left(a_{2}-a_{3}\right) c_{4}\right) x_{4}-\left(4 a_{4}-3 a_{5}+2 a_{6}\right) c_{4} x_{1}^{2} \\
& +\left(2 a_{4}-a_{5}\right) c_{4} x_{1} x_{2}-\left(a_{5}-2 a_{6}\right) c_{4} x_{1} x_{3}+2\left(\left(4 a_{4}-a_{6}\right) c_{2}\right. \\
& \left.-2\left(a_{4}-a_{5}+a_{6}\right) c_{4}\right) x_{1} x_{4}+\left(\left(2 a_{4}-a_{5}\right) c_{4}-\left(4 a_{4}+a_{5}\right) c_{2}\right) x_{2} x_{4} \\
& +\left(2\left(a_{5}+a_{6}\right)-\left(a_{1}+a_{3}\right) c_{3}+\left(2 a_{6}-a_{5}\right) c_{4}\right) x_{3} x_{4} \\
& +\left(\left(a_{5}-2 a_{6}\right) c_{4}-2\left(a_{5}+a_{6}\right) c_{2}\right) x_{4}^{2}
\end{aligned}
$$

and

$$
L_{1}=-\left(a_{2}-a_{3}\right)+\left(4 a_{4}-3 a_{5}+2 a_{6}\right) x_{1}-\left(2 a_{4}-a_{5}\right) x_{2}+\left(a_{5}-2 a_{6}\right) x_{3}-\left(a_{5}-2 a_{6}\right) x_{4} .
$$

We shall use these expressions later on in the proof of Lemma 2.9.

We go back now to system (1.4). Since it has only one Darboux polynomial and one polynomial first integral, if it has an exponential factor, then it must be of the form 
$\exp \left(f /\left(F^{n} Q\left(H_{1}\right)\right)\right)$, with $n \in \mathbb{N} \cup\{0\}$ and $Q \in \mathbb{C}\left[H_{1}\right]$. Next we prove that the expression of an exponential factor of this form cannot contain a power of $F$ in the denominator of the exponent.

Lemma 2.8. Suppose that system (1.4) has an exponential factor $E=\exp \left(f /\left(F^{n} Q\left(H_{1}\right)\right)\right.$, with $f \in \mathbb{C}\left[x_{1}, x_{2}, x_{3}, x_{4}, x_{5}\right], n \in \mathbb{N} \cup\{0\}, F \nmid f$ and $Q$ a polynomial. Then $n=0$.

Proof. Suppose that $n>0$. Let $L$ be the cofactor of $E$. Since $\mathcal{X}\left(Q\left(H_{1}\right)\right)=0$, we have

$$
L E=\mathcal{X}(E)=E \frac{\mathcal{X}(f) \cdot F^{n}-f \cdot \mathcal{X}\left(F^{n}\right)}{F^{2 n} Q\left(H_{1}\right)} .
$$

Hence

$$
\mathcal{X}(f) F^{n}+n c_{3} x_{4} f F^{n}=L F^{2 n} Q\left(H_{1}\right)
$$

see Lemma 2.4. Therefore

$$
\mathcal{X}(f)+n c_{3} x_{4} f=L F^{n} Q\left(H_{1}\right) .
$$

Since $n>0$, equation (2.13) on $H_{1}=h$ and $F=0$ becomes

$$
\begin{aligned}
& \left(-c_{1} x_{1} x_{2}^{2}-c_{4} x_{1}+\left(c_{2}-c_{4}\right) x_{4}+c_{4} h\right) \frac{\partial \bar{f}}{\partial x_{1}}+\left(-2 c_{1} x_{1} x_{2}^{2}-c_{4} x_{1}-c_{4} x_{4}+c_{4} h\right) \frac{\partial \bar{f}}{\partial x_{2}} \\
& \quad+\left(c_{1} x_{1} x_{2}^{2}-2 c_{2} x_{4}\right) \frac{\partial \bar{f}}{\partial x_{4}}=-n c_{3} x_{4} \bar{f} .
\end{aligned}
$$

where $\bar{f}$ is the restriction of $f$ to $H_{1}=h$ and $F=0$. This means that $\bar{f}$ is a Darboux polynomial of system (2.7) with cofactor $-n c_{3} x_{4} \neq 0$. In view of the proof of Lemma 2.5 this is a contradiction, which comes from the assumption $n \neq 0$. Therefore $n=0$ and the lemma follows.

The following result completes the proof of statement (c).

Lemma 2.9. Let $E=\exp \left(f / Q\left(H_{1}\right)\right)$ be an exponential factor of system (1.4), with $Q \in$ $\mathbb{C}\left[H_{1}\right]$ and $f \in \mathbb{C}\left[x_{1}, x_{2}, x_{3}, x_{4}, x_{5}\right]$. Then $f-g Q\left(H_{1}\right)$, with $g$ as in $(1.5)$, is a polynomial function of $H_{1}$.

Proof. Set $x_{5}=H_{1}-x_{1}-x_{4}$. We write the cofactor $k$ of $\exp \left(f / Q\left(H_{1}\right)\right)$ in the variables $x_{1}, x_{2}, x_{3}, x_{4}, H_{1}$ as follows:

$$
\begin{aligned}
k= & k_{0}+k_{1} x_{1}+k_{2} x_{2}+k_{3} x_{3}+k_{4} x_{4}+k_{5} x_{1}^{2}+k_{6} x_{1} x_{2}+k_{7} x_{1} x_{3}+k_{8} x_{1} x_{4} \\
& +k_{9} x_{2}^{2}+k_{10} x_{2} x_{3}+k_{11} x_{2} x_{4}+k_{12} x_{3}^{2}+k_{13} x_{3} x_{4}+k_{14} x_{4}^{2} \\
& +\left(k_{15}+k_{16} x_{1}+k_{17} x_{2}+k_{18} x_{3}+k_{19} x_{4}\right) H_{1}+k_{20} H_{1}^{2}
\end{aligned}
$$


where $k_{i} \in \mathbb{C}$ for all $i$. We also write $Q$ and $f$ as polynomials in $H_{1}$ :

$$
Q\left(H_{1}\right)=\sum_{j=0}^{n} d_{j} H_{1}^{j} \quad \text { and } \quad f=\sum_{j=0}^{n} f_{j}\left(x_{1}, x_{2}, x_{3}, x_{4}\right) H_{1}^{j},
$$

where $d_{j} \in \mathbb{C}$ and $f_{j} \in \mathbb{C}\left[x_{1}, x_{2}, x_{3}, x_{4}\right]$. Since $E$ is an exponential factor, $f$ satisfies

$$
\mathcal{X}(f)=k Q\left(H_{1}\right)
$$

Evaluating (2.14) on $H_{1}=0$, we have that $\exp \left(f_{0}\right)$, with $f_{0}=\left.f\right|_{H_{1}=0}$, is an exponential factor of system (2.6) with $h=0$ with the cofactor $d_{0} \bar{k}=\left.d_{0} k\right|_{H_{1}=0}$. In view of Lemma 2.6, we have $f_{0}=f_{0}^{0}+d_{0} g$, with $g$ as in (1.5). Moreover, $\bar{k}=L_{0}$. Now computing the coefficient of $H_{1}$ in (2.14) we get

$$
\begin{aligned}
c_{4} \frac{\partial f_{0}}{\partial x_{1}} & +c_{4} \frac{\partial f_{0}}{\partial x_{2}}+\left(-c_{1} x_{1} x_{2}^{2}-c_{4} x_{1}+\left(c_{2}-c_{4}\right) x_{4}\right) \frac{\partial f_{1}}{\partial x_{1}} \\
& +\left(-2 c_{1} x_{1} x_{2}^{2}-c_{4} x_{1}-c_{4} x_{4}\right) \frac{\partial f_{1}}{\partial x_{2}}+\left(c_{2}-c_{3} x_{3}\right) x_{4} \frac{\partial f_{1}}{\partial x_{3}} \\
& +\left(c_{1} x_{1} x_{2}^{2}-c_{2} x_{4}-c_{3} x_{3} x_{4}\right) \frac{\partial f_{1}}{\partial x_{4}} \\
& =d_{1} L_{0}+d_{0}\left(k_{15}+k_{16} x_{1}+k_{17} x_{2}+k_{18} x_{3}+k_{19} x_{4}\right) .
\end{aligned}
$$

Proceeding as in the proof of Lemma 2.6, we obtain $f_{1}=f_{1}^{0}+d_{1} g$ and $k_{15}+k_{16} x_{1}+$ $k_{17} x_{2}+k_{18} x_{3}+k_{19} x_{4}=c_{4} L_{1}$. Now computing the coefficient of $H_{1}^{2}$ in (2.14) we get

$$
\begin{aligned}
c_{4} \frac{\partial f_{1}}{\partial x_{1}} & +c_{4} \frac{\partial f_{1}}{\partial x_{2}}+\left(-c_{1} x_{1} x_{2}^{2}-c_{4} x_{1}+\left(c_{2}-c_{4}\right) x_{4}\right) \frac{\partial f_{2}}{\partial x_{1}} \\
& +\left(-2 c_{1} x_{1} x_{2}^{2}-c_{4} x_{1}-c_{4} x_{4}\right) \frac{\partial f_{2}}{\partial x_{2}}+\left(c_{2}-c_{3} x_{3}\right) x_{4} \frac{\partial f_{2}}{\partial x_{3}} \\
& +\left(c_{1} x_{1} x_{2}^{2}-c_{2} x_{4}-c_{3} x_{3} x_{4}\right) \frac{\partial f_{2}}{\partial x_{4}} \\
= & d_{2} L_{0}+d_{1} L_{1}+d_{0} k_{20},
\end{aligned}
$$

or equivalently

$$
\begin{aligned}
& \left(-c_{1} x_{1} x_{2}^{2}-c_{4} x_{1}+\left(c_{2}-c_{4}\right) x_{4}\right) \frac{\partial f_{2}}{\partial x_{1}}+\left(-2 c_{1} x_{1} x_{2}^{2}-c_{4} x_{1}-c_{4} x_{4}\right) \frac{\partial f_{2}}{\partial x_{2}} \\
& +\left(c_{2}-c_{3} x_{3}\right) x_{4} \frac{\partial f_{2}}{\partial x_{3}}+\left(c_{1} x_{1} x_{2}^{2}-c_{2} x_{4}-c_{3} x_{3} x_{4}\right) \frac{\partial f_{2}}{\partial x_{4}}=d_{2} L_{0}+d_{0} k_{20} .
\end{aligned}
$$

Proceeding again as in the proof of Lemma 2.6 we get $f_{2}=f_{2}^{0}+d_{2} g$ and $k_{20}=0$. Therefore $k=L$, see (2.12). Proceeding inductively with $k=L$ we get $f_{j}=f_{j}^{0}+d_{j} g$, 
for $j \geq 2$. In short,

$$
f=\sum_{j=0}^{n} d_{j}\left(f_{j}^{0}+g\right) H_{1}^{j}=P\left(H_{1}\right)+g Q\left(H_{1}\right),
$$

with $P\left(H_{1}\right)=\sum_{j=0}^{n} d_{j} f_{j}^{0} H_{1}^{j}$ and $g$ as in (1.5). Then the lemma follows.

After Lemma 2.9, if $\exp \left(f / Q\left(H_{1}\right)\right)$ is an exponential factor, then

$$
e^{f / Q\left(H_{1}\right)}=e^{g} e^{P\left(H_{1}\right) / Q\left(H_{1}\right)},
$$

with $P$ a polynomial in $H_{1}$. Then statement (c) follows.

Remark 2.10. The cofactors of $F_{1}, \ldots, F_{6}$ are, respectively,

$$
\begin{aligned}
& k_{1}=\left(c_{2}-c_{3} x_{3}\right) x_{4} ; \\
& k_{2}=-2 c_{2} x_{4}-c_{4} x_{5} ; \\
& k_{3}=-c_{3} x_{3} x_{4}+c_{4} x_{5} ; \\
& k_{4}=-2\left(-2 x_{1}+x_{2}\right)\left(2 c_{2} x_{4}+c_{4} x_{5}\right) ; \\
& k_{5}=-c_{2} x_{2} x_{4}+2 c_{2} x_{3} x_{4}-2 c_{2} x_{4}^{2}-3 c_{4} x_{1} x_{5}+c_{4} x_{2} x_{5}+c_{4} x_{3} x_{5}-c_{4} x_{4} x_{5} ; \\
& k_{6}=-2\left(x_{1}-x_{3}+x_{4}\right)\left(c_{2} x_{4}-c_{4} x_{5}\right) .
\end{aligned}
$$

\subsection{Proof of statement (d)}

Let $H$ be a Darboux first integral of system (1.4). Then it must be of the form $H=$ $F^{\lambda_{1}} \exp (g)$ where $g$ is given in (1.5). In view of Proposition 1.1, we must have

$$
\begin{aligned}
0 & =-\lambda_{1} c_{3} x_{4}+L \\
& =\left(\left(a_{1}-2 a_{2}\right) c_{2}-\lambda_{1} c_{3}\right) x_{4}+\left(a_{3}-a_{2}\right) c_{4} x_{5}+2\left(4 a_{4}-a_{6}\right) c_{2} x_{1} x_{4} \\
& +\left(4 a_{4}+3 a_{5}+2 a_{6}\right) c_{4} x_{1} x_{5}+\left(a_{5}-4 a_{4}\right) c_{2} x_{2} x_{4}-\left(2 a_{4}+a_{5}\right) c_{4} x_{2} x_{5} \\
& +\left(-2 a_{5} c_{2}+2 a_{6} c_{2}-a_{1} c_{3}-a_{3} c_{3}\right) x_{3} x_{4}-\left(a_{5}+2 a_{6}\right) c_{4} x_{3} x_{5} \\
& +2\left(a_{5}-a_{6}\right) c_{2} x_{4}^{2}+\left(a_{5}+2 a_{6}\right) c_{4} x_{4} x_{5},
\end{aligned}
$$

where $L$ is the cofactor of $\exp (g)$, see (2.12). Solving (2.15) we get $\lambda=3 a_{1} c_{2} / c_{3}, a_{2}=$ $a_{3}=-a_{1}$ and $a_{4}=a_{5}=a_{6}=0$. Therefore statement (d) follows.

Acknowledgments. A. Ferragut is partially supported by the Spanish Government grant MTM2013-40998-P and C.Valls is supported by Portuguese National Funds through FCT - Fundação para a Ciência e a Tecnologia within projects PTDC/MAT/117106/2010 and PEst-OE/EEI/LA0009/2013 (CAMGSD). 


\section{References}

[1] M. BANAJI, $P$ matrix properties, injectivity, and stability in chemical reaction systems, SIAM J. Appl. Math. 67 (2007), 1523-1547.

[2] M. BANAJI AND G. CRACIUN, Graph-theoretic approaches to injectivety and multiple equilibria in systems of interacting elements, Commun. Math. Sci. 7 (2009), 867-900.

[3] M. Banaji And G. Craciun, Graph-theoretic approaches for injectivety and unique equilibria in general chemical reaction systems, Adv. Appl. Math. 44 (2010), 168-184.

[4] G. Craciun And M. Feinberg, Multiple equilibria in complex chemical reaction networks I: The injectivity property, SIAM J. Appl. Math. 65 (2005), 1526-1546.

[5] G. Craciun and M. Feinberg, Multiple equilibria in complex chemical reaction networks II: The species reaction graph, SIAM J. Appl. Math. 66 (2006), 1321-1338.

[6] G. Craciun and M. Feinberg, Multiple equilibria in complex chemical reaction networks: Semiopen mass action systems, SIAM J. Appl. Math. 70 (2010), 18591877.

[7] F. Dumortier, J. Llibre And J.C. ARTÉs, Qualitative theory of planar differential systems, Universitext, Springer, New York, 2004.

[8] E. Feliu, C. WiUf, Simplifying biochemical models with intermediate species, J. R. Soc. Interface 10 (2013), 20130484.

[9] E. Feliu, C. WiUf, Preclusion of switch behavior in networks with mass-action kinetics, Appl. Math. Comput. 219 (2012), 1449-1467.

[10] E. Feliu, C. WiUf, Variable Elimination in Chemical Reaction Networks with MassAction Kinetics, SIAM J. Appl. Math., 72(4) (2012), 959-981.

[11] J. Llibre, X. Zhang, On the Darboux Integrability of Polynomial Differential Systems, Qual. Theory Dyn. Syst. 11 (2012), 129-144. 